\title{
Real-Time 100 Gb/s NRZ-OOK Transmission with a Silicon Photonics GeSi Electro-Absorption Modulator
}

\author{
J. Verbist ${ }^{1,2^{*}}$, M. Verplaetse ${ }^{1}$, S. A. Srinivasan ${ }^{2,4}$, P. De Heyn ${ }^{4}$, T. De Keulenaer ${ }^{1,3}$, R. Vaernewyck ${ }^{1,3}$, \\ R. Pierco ${ }^{1,3}$, A. Vyncke ${ }^{1,3}$, P. Verheyen ${ }^{4}$, S. Balakrishnan ${ }^{4}$, G. Lepage ${ }^{4}$, M. Pantouvaki ${ }^{4}$, P. Absil ${ }^{4}$, \\ X. Yin ${ }^{1}$, G. Roelkens ${ }^{2}$, G. Torfs ${ }^{1}$, J. Van Campenhout ${ }^{4}$, J. Bauwelinck ${ }^{1}$ \\ ${ }^{1}$ IDLab, INTEC, Ghent University - imec, 9052 Ghent, Belgium \\ ${ }^{2}$ Photonics Research Group, INTEC, Ghent University-imec, 9052 Ghent, Belgium \\ ${ }^{3}$ BiFast, Spin-off incubation project of Ghent University-imec, IDLab, 9052 Ghent, Belgium \\ ${ }^{4}$ imec, Kapeldreef 75, 3001 Leuven, Belgium \\ *jochem.verbist@ugent.be
}

\begin{abstract}
We demonstrate single-wavelength, serial and real-time $100 \mathrm{~Gb} / \mathrm{s}$ NRZ-OOK transmission over $500 \mathrm{~m}$ SSMF with a GeSi EAM implemented on a silicon photonics platform. The device was driven with $2 \mathrm{Vpp}$ without $50 \Omega$ termination, allowing a low-complexity solution for $400 \mathrm{GbE}$ short-reach optical interconnects.
\end{abstract}

\section{Introduction}

The increasing growth of internet traffic pushes the requirements on the intra-datacenter high-speed optical interconnects. This has led to the evolution from $100 \mathrm{~Gb} / \mathrm{s}$ Ethernet to $400 \mathrm{~Gb} / \mathrm{s}$ Ethernet, for which the implementation options are currently under discussion [1]. A four lane $100 \mathrm{~Gb} / \mathrm{s}$ scheme could be a relatively simple approach to achieve this goal allowing lower lane counts and as such, a higher spatial efficiency. Previously, $100 \mathrm{~Gb} / \mathrm{s}$ single-lane transmissions have been realized using PAM-4, discrete multi-tone (DMT), and electrical duobinary (EDB), but most of these demonstrations relied on heavy off-line digital signal processing (DSP) [2-5]. $112 \mathrm{~Gb} / \mathrm{s}$ PAM-4 modulation of a discrete Mach-Zehnder modulator at 8.6W power consumption was demonstrated in [6]. In [7] EDB was used at $100 \mathrm{~Gb} / \mathrm{s}$ in combination with a travelling-wave electro-absorption modulator with integrated DFB-laser in an InP photonic integrated circuit and in [8] $100 \mathrm{~Gb} / \mathrm{s}$ non-real-time OOK transmission was achieved through a siliconorganic-hybrid modulator. Although the required drive voltages in [8] and [10] are comparable to our experiment, the transmission line structure of the modulator electrode necessitates a power-consuming $50 \Omega$ termination. Here we present $100 \mathrm{~Gb} / \mathrm{s}$ NRZ-OOK transmission of a compact, lumped Germanium-Silicon EAM without $50 \Omega$ termination integrated on a silicon photonics platform, in combination with an in-house designed transmitter (TX) and receiver (RX) chipset in a SiGe BiCMOS technology. This is the first real-time $100 \mathrm{~Gb} / \mathrm{s}$ NRZ-OOK transmission with a silicon-based modulator without any DSP.

\section{Experiment Setup}

The EAM was fabricated in imec's $200 \mathrm{~mm}$ silicon photonics platform and consists of an $80 \mu \mathrm{m}$ long and $600 \mathrm{~nm}$ wide germanium waveguide with embedded lateral p-i-n junction, connected to silicon waveguides. The operation is based on the Franz-Keldish effect, which shifts the band edge of Ge by applying an electrical field [9]. Light is coupled in and out of the waveguide structure through fiber-to-chip grating couplers (IL $=\sim 6 \mathrm{~dB} /$ coupler). Electrical RF probes, without any termination, are used to apply the high-speed signal and the bias voltage to the EAM. Figure 1 shows the schematic of the setup for the transmission experiments. Four individual $2^{7}-1$ long pseudo-random bitstreams (PRBS) are generated at $25 \mathrm{~Gb} / \mathrm{s}$ on a XilinX FPGA-board and multiplexed with the required delays to form again a $2^{7}-1$ long PRBS at $100 \mathrm{~Gb} / \mathrm{s}$. An analog six-tap feedforward equalizer (FFE) on the transmitter IC is used to compensate frequency roll-off and non-idealities of the following components and the link. A RF amplifier with internal bias- $\mathrm{T}$ delivers a swing of $\sim 2 \mathrm{Vpp}$ to the modulator.

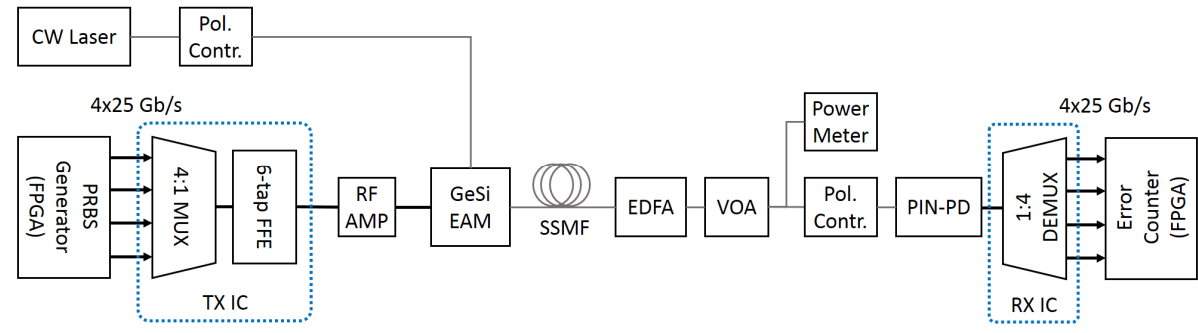

Figure 1. Experimental setup 
The EAM was biased at $-1.85 \mathrm{~V}$. The waveguide-coupled laser power was $7.5 \mathrm{dBm}$ at a wavelength of $1601.5 \mathrm{~nm}$. With these settings we estimated a dynamic extinction ratio of $\sim 6.5 \mathrm{~dB}$ and an insertion loss of $\sim 7 \mathrm{~dB}$ for the modulator. No temperature control was used during the experiments. As no high-speed transimpedance amplifier with sufficient bandwidth was available, we had to increase the signal power with an EDFA before coupling to a commercial PINphotodiode $(\mathrm{BW}=50 \mathrm{GHz})$. Finally, the receiver IC deserializes the $100 \mathrm{~Gb} / \mathrm{s}$ signal into $4 \mathrm{x} 25 \mathrm{~Gb} / \mathrm{s}$ streams and provides these to the FPGA for real-time error detection without the need for complex DSP. The TX and RX ICs were designed in-house in a $0.13 \mu \mathrm{m}$ BiCMOS technology [7, 10]. The TX IC occupies $\sim 1.5 \mathrm{~mm} \times 4.5 \mathrm{~mm}$ and consumes $<1 \mathrm{~W}$. The RX IC occupies $\sim 2 \mathrm{~mm} \times 2.5 \mathrm{~mm}$ and uses less than $1.2 \mathrm{~W}$.

\section{Results and Discussion}

Optical performance of the link was verified using the setup shown in Fig. 1. At a rate of $100 \mathrm{~Gb} / \mathrm{s} \mathrm{NRZ}$ almost all the components in the E/O/E-link (65 GHz RF Amp, $50 \mathrm{GHz}$ PIN-PD, cables and connectors, fiber dispersion,...) influence the overall response in the frequency range of interest. The frequency response of the optical link (from RF Amp to PIN-PD) for different lengths of fiber can be seen in Fig. 2 (b). We clearly see that standard single mode fiber (SSMF) operating in L-band (1601.5nm) severely degrades the flatness of the frequency response at longer fiber spans. Nevertheless, we still manage to obtain bit-error ratios (BER) comfortably under the hard-decision forward error coding limit of $3.8 \times 10^{-3}$ (HD-FEC with $7 \%$ overhead) over $500 \mathrm{~m}$ of SSMF as shown in Fig. 2 (a). Investigating the eye diagrams in Fig. 2 (c) and (d) for B2B and 500m transmission captured directly after the PIN-PD with a $70 \mathrm{GHz}$ sampling scope, we believe that the BW of the RX IC (41 GHz) is the most significant contributor to the total BW, limiting the overall link performance. Tests with a new and faster version of the RX IC are planned.
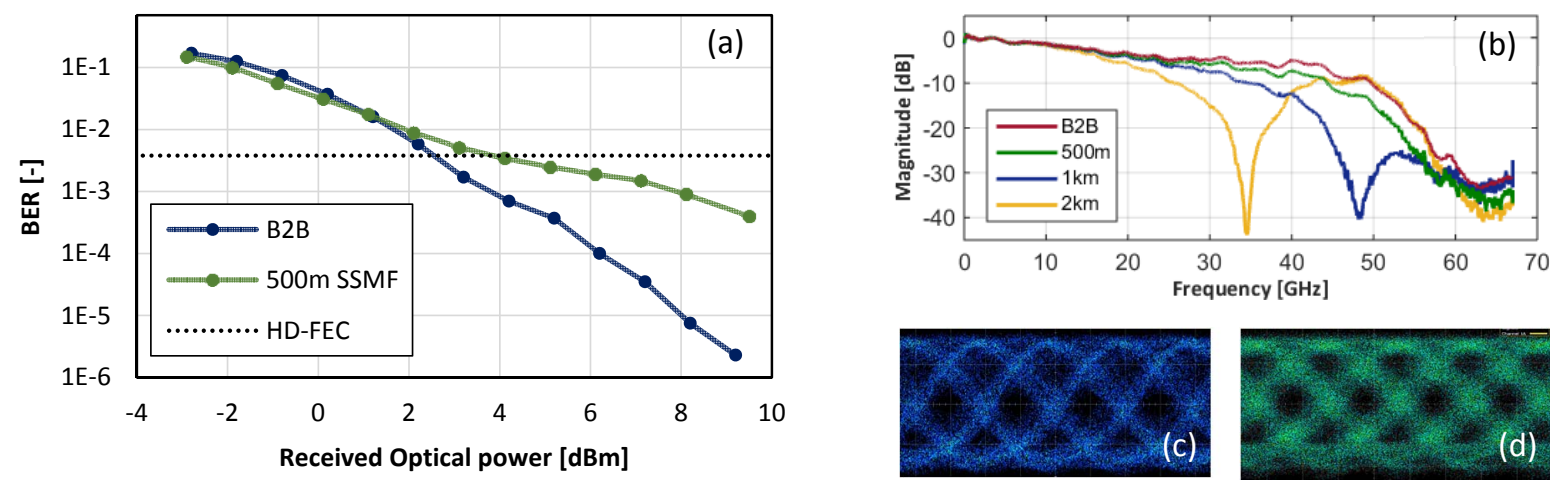

Figure 2. (a) Real-time BER curves for B2B and $500 \mathrm{~m}$ fiber transmission; (b) Frequency response of the optical link (from RF Amp to PIN-PD) for different lengths of SSMF at $1601.5 \mathrm{~nm}$; Captured $100 \mathrm{~Gb} / \mathrm{s} \mathrm{NRZ}$ eyes from photodiode (c) back-to-back and (d) after 500m SSMF fiber.

\section{Conclusion}

We have demonstrated a GeSi EAM fabricated in a $200 \mathrm{~mm}$ silicon photonics platform that is capable of transmitting $100 \mathrm{~Gb} / \mathrm{s} \mathrm{NRZ}$ in combination with an in-house designed SiGe BiCMOS transmitter and receiver chipset. Successful transmission over $500 \mathrm{~m}$ of SSMF was achieved and verified in real-time without any offline DSP, paving the way for a compact, low-complexity silicon photonic transceivers for $400 \mathrm{GbE}$ short-reach optical interconnects.

\section{Acknowledgement}

This work was supported by the imec IAP on Optical I/O for the Ge EAM, the UGent IOF for the Tx/Rx ICs, the H2020 TERABOARD project, the UGent BOF and the FWO.

\section{References}

[1] "IEEE P802.3bs 400 Gigabit Ethernet Task Force." [Online]. Available: http://www.ieee802.org/3/bs/

[2] C. Yang et al, IEEE Photonics Journal, vol. 8, no. 3, 2016.

[3] K. Zhong et al, Optics Express, vol. 23, no. 2, pp. 1176-1189, 2015.

[4] D. Sadot et al, in Optical Networking and Communication Conf., Los Angeles, 2015

[5] J. Lee et al, in Optical Networking and Communication Conf., San Francisco, 2014.
[6] J. Lee et al, in ECOC, ID:0604, Valencia, 2015.

[7] M. Verplaetse et al., Journ. of Lightw. Techn., (2017), to be published

[8] W. Hartmann et al., in ECOC, ID:1078, Valencia (2015)

[9] S. A. Srinivasan et al, OFC, Anaheim, CA, 2016, pp. 1-3.

[10] T. De Keulenaer et al., Electronics Letters, vol. 51, no. 4, pp. 343-345, 2015 